\title{
Face-to-Face Sociability Signs Made Explicit in CMC
}

\author{
Carla Faria Leitão, Clarisse Sieckenius de Souza, and Clarissa Maria de A. Barbosa \\ SERG - The Semiotic Engineering Research Group, Informática, PUC-Rio \\ Rua Marquês de São Vicente, 225, Gávea, 22453-900, Rio de Janeiro, RJ, Brazil \\ \{cfaria, clarisse, cbarbosa\} @inf.puc-rio.br
}

\begin{abstract}
This paper discusses how semiotic engineering can support the formulation of problems and solutions involved in handling face-to-face (F2F) sociability models in computer-mediated communication (CMC). Based on a case study where a group of users migrated through different types of CMC systems, we show that the designer's model of F2F sociability is extensively signified and encoded into technology, whether they know it or not. Users are deeply affected by the designers' F2F sociability models. Two qualitative methods of analysis are used to reveal the richness of interpretive and communicative processes in which online communities are involved, and the interplay of designers' and users' signs at interaction time.
\end{abstract}

Keywords: Semiotic engineering; CMC; Sociability models.

\section{Introduction}

Nearly two decades past the dissemination of Information and Communication Technology, the fears about the dissolution of social ties [1] are not as threatening as they used to be. Computer-mediated communication (CMC) has allowed users to complement their face-to-face sociability. Nowadays, far from being extinguished by technology, F2F relationships constitute the reference model for online relationships. Both designers ${ }^{1}$ and users leave the imprint of their social values on the technology that they produce and adopt [3]. The integration of the values related to F2F forms of interaction constitutes a face-to-face sociability model (F2F model).

This paper sets out to present the contribution of semiotic engineering [6], a semiotically-inspired theory of human-computer interaction (HCI), to the study of some of the problems involved in migrating F2F communication practices into CMC environments. Most current research into this migration concentrates on users, their expectations and experience. Although a user-centered perspective is fundamental to $\mathrm{HCI}$, of no less importance is a perspective that centers on designers, and on how they project their values and vision onto the technology they build.

In semiotic engineering, the main function of computer systems interfaces is to bring designers and users together at interaction time, and to communicate the designers' vision to users through computer-human interaction. This vision carries not

${ }^{1}$ In this paper, the word designer(s) will be used to refer not only to those that specifically 'design' technology, but also to those that develop and produce it. 
only the design rationale (i.e. the 'why', 'what for', and 'how to' of technology), but also the subjective and cultural values that guide design choices. Therefore, interface signs always communicate elements of the designers' F2F model to CMC users. Nonetheless, the sense users will make of such signs is largely unpredictable.

In order to illustrate how F2F model communication is achieved, and how it can affect users and their own sociability models, we present a case study. The specific context of the study is especially significant. Firstly, this is primarily a group of multiple sclerosis (MS) sufferers, with the occasional participation of family members and care-takers. MS is a seriously debilitating disease, and sufferers often have considerable difficulty to move about and socialize in diversified physical settings. As a result, for some, CMC may be the only opportunity to socialize with a group, representing a radical movement from $\mathrm{F} 2 \mathrm{~F}$ sociability to online interactions. Secondly, this group (or "SPEM" - Sociedade dos Portadores de Esclerose Múltipla ${ }^{2}$ ) is a successful one. The duration of the group (approximately 5 years by now), the volume of messages (over 14000), the number of members (200 to 500 members, approximately, at different points in time), and messages exchanged by members explicitly alluding to the fact that they are a successful online group are all good evidence of their success. Thirdly, the study covered a period of technological migration. SPEM had been using a particular kind of technology till January 2004, but then they were forced to move to another kind of technology because of problems experienced with their Internet Service Provider. Together, these contextual factors allowed us to take a deep perspective into some of the hard challenges brought about by technology designed to support CMC.

The SPEM case study led us to the following conclusions:

1. Groupware designers, whether they know it or not, imprint in the systems they design cultural and personal values that emerge from their F2F models of groups.

2. The force of cultural values shared by community members constantly (re)introduces signs of such values, in practice. When they are contradictory with those imposed by design, signs of conflict or ambiguity can be found.

The remainder of this paper is organized in four additional sections. In section 2 , we present the gist of semiotic engineering, providing the necessary concepts for the reader to appreciate our discussions. Then, in section 3 we briefly present two models of F2F sociability, originating in classical sociology. Based on these two models and on the ontology of semiotic engineering, we present our case study in section 4 . Section 5 wraps up the case with our discussions and conclusions.

\section{A Semiotic Theory of HCI}

Semiotic engineering [6] is a theory of HCI, whose ontology is rooted in such fundamental semiotic concepts as: sign, signification, communication, and interpretation. The first and last concepts stem from Peirce's semiotics [14]; the other two are inspired by Eco's theory of semiotics [9]. The aim of semiotic engineering is not the same as any of these two theories - we do not seek to investigate signs,

\footnotetext{
${ }^{2}$ Multiple Sclerosis Sufferers Society.
} 
signification, communication or interpretation processes per se. We seek to investigate human-computer interaction, a well-defined object of study, where human and technological components are brought together to form an indivisible whole.

The foundational concepts that support the semiotic engineering ontology are:

1. A sign is anything that is taken to mean something, by some mind (human or not), in one respect or another [14]. This very broad definition bears some interesting implications. For example, it implies that signs don't have to be consensual. Of course there are many kinds of signs: some are cultural conventions, some are produced and processed by humans alone, and others may be produced and processed by machines. According to Peirce, the human species is biologically equipped to constantly produce and exchange an unlimited range of signs.

2. Signification is the process by which certain expressions are systematically associated with certain contents based on culturally established conventions [9]. This process establishes a variety of sign systems in every culture - verbal and non-verbal, limited or unlimited, and so on. The units of signification systems are signs.

3. Communication is the process by which people use signs and explore the possibilities of signification systems to achieve an unlimited range of purposes [9]. Inasmuch as communication is structured in terms of well established and contextualized signs, recipients and interpreters are likely to get the communicator's message with relatively little effort. However, for communicators, the available signification systems are a resource, and not a limitation. They can easily twist the system (as in jokes), use it creatively (as in metaphors), and even expand it (as in neologisms). Because receivers and interpreters are equally apt to twisting, adapting and expanding culturally-determined signification systems, such steps outside their limits are most frequently successful, and often go unnoticed.

4. Interpretation, in semiotic engineering, is aligned to the notion of semiosis [14]. The sign is structured by the mutual relations of three components: the representation of the sign, the object to which the representation refers, and the particular interpretation by virtue of which representation and object are bounded. The relation between a sign representation and its object, or referent, is mediated. Moreover, the mediating interpretation that relates representation to referent is not a value, but a process - called semiosis. Semiosis unfolds over unlimited time and space - it is not a finite calculation producing one definitive value.

The implications of the concepts above reach far, and shed new light on HCI. The very possibility of taking anything as a potential sign (from 1), and the unlimited nature of semiosis (from 4), explains why communication (from 3) can be successful. Interlocutors 'make up' signs of their own, or use known signs in completely novel ways, but since their interpreters have exactly the same abilities, communication is based on but not limited to the systematic associations of expression and content, conventionalized by culture. Therefore, in this perspective, it is not possible to capture or predict the meaning of any sign - whatever correspondence is made between expression (a sign) and content (a meaning, or range of meanings) involves a selection of some interpretations to the detriment of others.

As a result, humans often communicate without sharing the same meanings. It is only when counter factual evidence of their mutual understanding arises that they 
revise their meanings. A key resource for revising meanings in communication is 'metacommunication' - communication about communication. This pervasive resource encourages us to precipitate our conclusions about what this or that sign means - we reckon that if we are wrong, some other signs will give the evidence of our misunderstanding, and our interlocutors will correct us. For as long as they do not correct us, we assume that mutual understanding is in place. Mutual understanding depends on the ability and disposition of interlocutors to engage in learning and discovery. In conversation, speakers and listeners control semiosis by halting and resuming the ongoing interpretive process when they have evidence of successful and unsuccessful hypotheses, respectively. In some cases, this kind of control can be costly in emotional and social terms.

In the HCI scenario, systematic research about the social [8] or the emotional [13] [15] aspects of computing has been gaining momentum in recent years. In all these cases, however, theories and approaches have resorted to different ontologies when they talk about design time and interaction time. Such is not the case of semiotic engineering, and some important consequences of integrating design and interaction under the same ontology naturally follow.

Semiotic engineering views HCI as a particular case of CMC. In it, computer systems are defined as a one-shot designer-to-user message communicating the design intent, the designer's understanding of users' needs and aspirations, the vision of how the designed artifact will affect the users' lives, and how the artifact should be used to achieve its ultimate purpose. The message can be paraphrased as follows [6]:

"Here is my understanding of who you are, what I've learned you want or need to do, in which preferred ways, and why. And this is the system that I have therefore designed for you, and the way you can or should use it to fulfill a range of purposes that fall within this vision"

The metacommunication message gradually unfolds as users interact with the artifact and, in the process of semiosis, build their own interpretation of what the artifact means. The various meanings exchanged in successive turns of interaction contribute to the global sense-making process by which users appropriate the artifact as an achieved sign. Through metacommunication, the designer is present at interaction time. However, not physically present - the designer's communication about what he or she meant to communicate, and about how users can or should, themselves, communicate with the artifact, is delivered through the user interface. In semiotic engineering, the user interface is the designer's deputy at interaction time. The communication codes for user-system interaction do not need to be verbal. Direct manipulation interfaces, for example, privilege visual codes. However, explicit metacommunication messages (such as explanations, instructions) take verbal form.

The main difference introduced by semiotic engineering compared to existing theories of $\mathrm{HCI}$ is thus to frame the whole process of software production and software use as a homogeneous case of relayed communication. A similar idea was discussed by Winograd and Flores [18] two decades ago. Although the authors were proposing new foundations for software design, their work did not cover the details of how and why all layers of linguistic representation in computing are built, related, and eventually interpreted and used in all sorts of applications. They concentrated on computer-supported collaborative work contexts, and used speech act theory [17] to 
inform design decisions. Semiotics, however, provides a much broader and more general range of concepts than speech act theory. Humans and their computer counterparts are engaged in semiosis. The same ontological status of 'interlocutor' is conferred to users, designers and systems. As a result, an unbroken thread of concepts can be used to trace meanings in software production and software use.

The kinds of tools semiotic engineering provides for scientific research and professional practice in HCI are inspired by Schön's reflection-in-action perspective [16]. They are epistemic tools. They are meant to guide the naming and framing stages of design problems. In agreement with semiotic processes, Schön's perspective on design is that every design activity is unique. Thus the most important knowledge it requires is an epistemology of practice. Instead of knowledge about solutions for known categories of problems, an epistemology of practice is knowledge about methods and interpretive frameworks to be used in understanding, categorizing, and solving an unlimited range of HCI problems. Hence, the epistemic tools proposed by semiotic engineering aim at improving and expanding the researchers' and designers' knowledge about problems and solutions in design.

One such epistemic tool is the interpretive schema embedded in the metacommuni cation message from designer-to-user. For the specific category of multi-user applications, the message is extended in order to include crucial meaning elements related to how computer-mediated communication among users is designed to take place. The message content can be paraphrased as follows [6]

"Here is my understanding of who you are, what I've learned you want or need to do, in which preferred ways, and why. And this is the system that I have therefore designed for you, and the way you can or should use it to fulfill a range of purposes that fall within this vision. You can communicate and interact with other users through the system. During communication, the system will help you check:

Who is speaking? To whom?

What is the speaker saying? Using which code and medium? Are code and medium appropriate for the situation? Are there alternatives?

Is(are) the listener(s) receiving the message? What if not?

How can the listener(s) respond to the speaker?

Is there recourse if the speaker realizes the listener(s) misunderstood the message? What is it?"

Notice that by trying to elaborate the instantiation of this message for any CMC application, a designer is naturally led to reflect on expressive, semantic, pragmatic, and intentional aspects of communication.

Designers and researchers can use other semiotic engineering models and methods to gain deeper insight into the signs and signification systems that are used to support the users' interaction. One such method has been used in our case study - the semiotic inspection method [5][7]. The aim of the study was to find out how two classical F2F models were encoded in CMC technology used by SPEM members, and how users reacted to them.

\section{From Face-to-Face to Online Sociability Models}

A community is a key sociological concept, classically defined in opposition to society. In spite of much variety in perspective, there are some recurring ideas in the 
traditional studies of communities. Communities have been characterized as a group of people who experience a high degree of personal intimacy and emotional depth. They are self-sufficient, in that members can satisfy all their needs within the space defined by their mutual relations and collective values. Each member's identity is constructed in terms of the community itself. Social relations clearly define boundaries, unify the group, and provide protection against the threats of dissolution brought about by diverse and open-ended relations in a society [2] [12].

By opposition, society is defined by the multiple social roles played by each member, and the varying degrees of intimacy and emotional depth that they achieve in their relationships. To live in society is to have multiple sources for building one's identity, and to experience different kinds of belonging. It also implies the ability to live in and with various open groups, with multiple intersections with each other [12].

There is commonality between the notions of society and community: in classical sociology, they are both reference models for F2F relationships. However, early CMC environments were clearly inspired by the F2F community model [3]. Later, the original spirit was left way behind. Side by side with community models, other models sprang up, with very different sociability references, this time closer to the traditional concept of society than to that of community. A full-blown cultural and social technology-enabled phenomenon, the Internet welcomed developers and users who no longer sought unity, homogeneity, and group cohesion. They sought egocentered and multiple forms of interaction, based on both strong and weak ties, and motivated by individual needs [3]. Inspired by an F2F society model, online interactions became open, flexible, heterogeneous and diverse. Now both community and society models coexist and dictate the development of the Internet [3].

In order to further the discussion about the role of F2F models in the development and deployment of CMC, we now describe our case study, where different types of technologies were used, and their sociability models affected users in different ways.

\section{The Semiotic Engineering Account of SPEM}

The object of this study is a long-standing online group, the Multiple Sclerosis Sufferers Society, whose members are mainly MS sufferers and their families. The community was closely observed from October 2003 to March 2004, during which period the community migrated from one setting to another. This migration was a prime opportunity for us to detect some of the impacts of technology on groups. Then, in order to update our initial analysis of such impacts and verify its medium-term effects, we revisited and analyzed the community in March 2006 and in January 2007.

A combination of two qualitative methods was used in these analyses: the semiotic inspection method [5] [7] and the Underlying Discourse Unveiling Method (UDUM) [11]. Just like other qualitative methods, semiotic inspection and UDUM are nonpredictive, exploratory, and interpretive procedures that seek to expand the researcher's understanding of the study domain, based on the recurrence of meanings [4]. Semiotic inspection seeks implicit signification of design signs. UDUM, however, seeks to make the latent meanings present in the users' discourse explicit. By comparing results achieved by both methods, we set out to identify breakdowns and inconsistencies among design intent and the users' experience. 
Semiotic inspection should help the analyst reconstruct the designer-to-user message, and detect opportunities for breakdowns in communication. The inspection proceeds in five steps. In the first step, the analyst examines online and offline documentation and help content. In the second, the analyst examines static signs in the system. Static signs are those that appear on individual system screens, and are typically part of what designers refer to as interface layout. In the third, the analyst examines dynamic signs. These are signs that result from interaction, and include important meanings such as the causal effects of activating the various controls on the interface. In the fourth step, the analyst makes a contrastive comparison of designerto-user metacommunications identified in previous steps. Finally, in the fifth step, the analyst is able to produce a conclusive appreciation of the quality of the overall designer-to-user meta-communication.

UDUM complements the semiotic inspection by providing an analysis of natural language discourse produced by system users during interaction. The analysis is carried out in an iterated two-step procedure [11]. The inter-participant step examines the discourse of all users participating in the community's discussions. Identified recurring categories in what they say show the main trends in the group's thoughts. The intra-participant step examines the discourse of each single user, highlighting occasional inconsistencies and identifying the elaboration of meanings across all his or her interventions. The iteration of inter- and intra-participant analyses contributes to a very detailed characterization of what is actually being said.

The major findings of the combined analysis are grouped in four categories: the history of SPEM in cyberspace; the designers F2F sociability models; the users F2F sociability models; and designers and users together at interaction time.

\subsection{The History of SPEM in Cyberspace}

By January 2007, with approximately five years of existence, SPEM had 208 members and a repository of over 14.000 messages. By March 2004, however, SPEM had nearly 500 members, who had exchanged messages in three different discussion forums: InForum, MSN Groups and Yahoo!Groups. The role of each of these is made explicit in each one of the three environments, as well as on the group's homepage ${ }^{3}$. In addition to pointing to the other forums, the group owner introduces himself, and provides some information about multiple sclerosis. He also reveals the main purpose of SPEM: the exchange of experiences.

The history of SPEM shows three distinct eras, determined by the technology that the group was using. Period 1 (10/2003-01/2004) corresponds to the use of two different forums: InForum, used only for exchanging textual messages, and MSN Groups, for both textual communication and photo exchanges. MSN also provided support for chatting online, which a number of members used to do. Period 2 (02/2004-03/2004) corresponds to the use of three different forums. By this time, due to technical problems with InForum, the group owner decided to abandon the main forum and migrate to Yahoo!Groups. For one month, SPEM members we confused by using all three technologies. Finally, in Period 3 (04/2004 onwards) the group migrates to Yahoo!Groups, and chooses this environment to be the locale for all group activities. Figure 1 presents the distribution of the group over time and space.

\footnotetext{
${ }^{3} \mathrm{http}: / /$ marcelomorita.net/em
} 


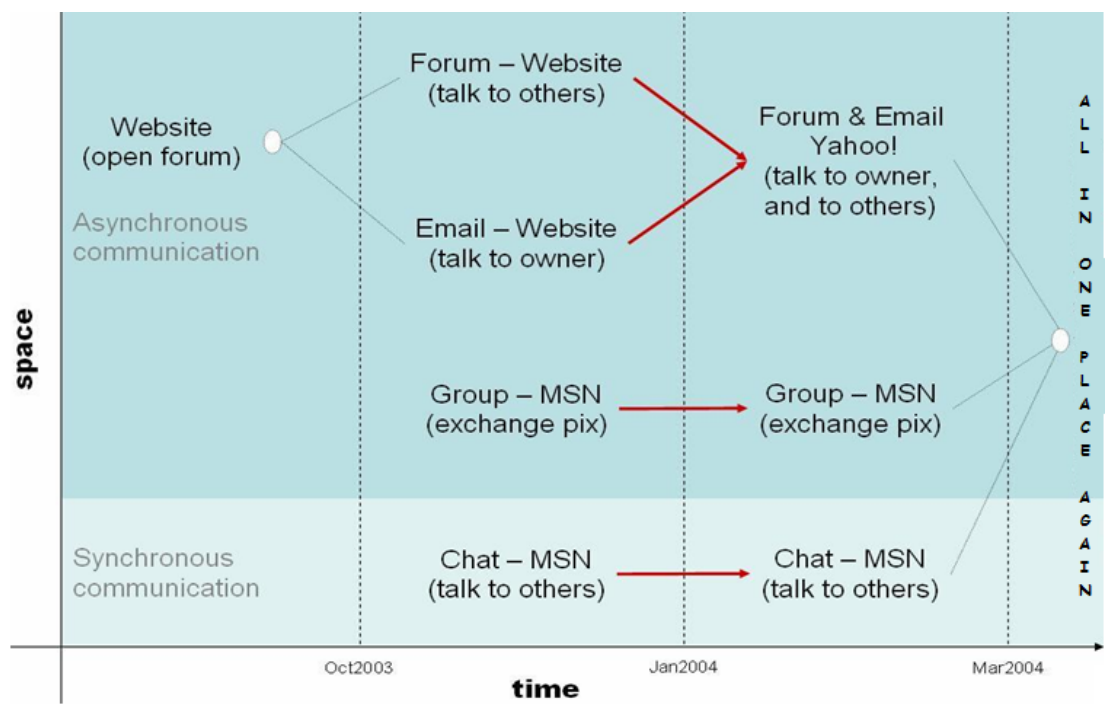

Fig. 1. SPEM community history - transitioning communicative locales

\subsection{The Designers' Face-to-Face Sociability Models}

The goal of semiotic inspection is to recover the overall metacommunication message of the designers of online forums used by SPEM. Designers signs allowed us to focus on one particular aspect of the metacommunication message, namely the one that says: "Here is my understanding of who you are, what I've learned you want or need to do, in which preferred ways, and why. And this is the system that I have therefore designed for you, and the way you can or should use it to fulfill a range of purposes that fall within this vision." Through the recovery of signs and meanings involved in this portion of the message, we have made explicit the F2F model of each technology.

InForum is a non-structured bulletin board, where messages appear in sequence, one after the other, with no threading. This is an online space with shallow verbal communication facilities, available to anyone interested in reading messages and posting material for users from all over the Internet to see. Messages are all presented in the same format, one after the other, in reverse chronological order. There are no signs indicating the number of participants, neither an indication of privacy policies. There is no way to register members (anyone is a member who wants to $\mathrm{read} / \mathrm{search} /$ post messages), and there can be no role differentiation among members. Members' presence can only be traced through message-posting, because it is only when composing a message that a member's identity is captured for other forum members to see. InForum does not support posting of pictures and other media files; neither does it support synchronous communication. The designers' metacommunication message in this case shows that the design vision was based on a society $\mathrm{F} 2 \mathrm{~F}$ model. This part of the metacommunication message can be paraphrased as this: "Here is an open space for socialization. Anyone can participate, by reading messages posted by others, only, or by posting messages, too. These messages are public in the Internet. Just like in offline society, you can choose different spaces to 
socialize. This is one such space, where all you can do is to read and exchange asynchronous text messages. If you wish other kinds of resources, I encourage you to find them on the Net, and to publish the appropriate links in here."

MSN Groups and Yahoo!Groups metacommunication is much more sophisticated than InForum's. They look very much alike, and their designers' signs communicate the same F2F model: that of a community. In both systems, group members must register and obtain a group ID. Thus, when they log in, they appear in the members list on the group's homepage, where the designers also tell users about how many members there are in this particular group, and what are the group configuration features. One such configuration (chosen by the group owner) is that, after registering and getting an ID, group members must wait for the moderator to accept their registration. Both systems allow group owners to choose whether they wish the group messages to be public on the Internet or not, as well as other privacy protection alternatives. MSN Groups and Yahoo!Groups both offer sophisticated chat tools. Messages can be viewed in different ways, and members can attach smileys to messages in order to express their mood. Texts and photos posted by members are stored in specifically structured spaces. Finally, other tools to coordinate activities, like a database, search mechanisms and alternative visualization forms, enable members to engage into complex goal-oriented activities. MSN Groups and Yahoo!'Groups metacommunication message can be paraphrased as this: "Here is a place for you to create an online community. In these communities all members are identified, even if they keep silent and don't take initiative in socializing activities. You can choose who you want to have in this community, and who you don't. I offer you useful communication and coordination resources. All you need to keep your community going is here, and you are protected against the outside world."

Having identified the designers' F2F models, let us see how these models were interpreted and appropriated by SPEM users them.

\subsection{The Users' Face-to-Face Sociability Models}

The UDUM-based analysis of members' discourse allows us to identify their underlying sociability models over the three periods of SPEM's existence.

In Period 1, when InForum and MSN Groups were both used, the content of messages exchanged in InForum was predominantly related to, in the group owner's own words, "making friends and talking to MS sufferers". Members would almost never talk about the technology they were using, and - notice this - they would always talk about themselves as a society, as their very name said: the Multiple Sclerosis Sufferers Society. The 'community' sign was never used to characterize SPEM. When using MSN Groups for chatting and posting pictures, all they needed to do was to get an MSN ID and log into the group's page (the group had declined the possibility offered by designers of having every registration request be authorized by the moderator). Occasionally, members would post text messages in the forum, but InForum was always referred to as the preferred place for this sort of communication. See what the message below expresses ${ }^{4}$.

${ }^{4}$ Transcribed messages were posted originally in Portuguese and translated by the authors, keeping up with the original writing style. 
"I am a bit late in welcoming you. (...) I'd like to ask you to go to www.inforum.insite.com.br. There you will find the same people who are here, and I also believe that things move much faster there."

MSN Groups was defined by a member as "a branch of SPEM". Introduced some time after Inforum, MSN Groups was easily incorporated by SPEM members. Resistance to use the new technology was not observed, since there were no messages about difficulties generated by the introduction of a more complex tool. Thus, there were no signs that social experiences were not negatively affected by the introduction of a new technology. MSN Groups users seemed to ignore the MSN designers' metacommunication message, tacitly declining most of the designers' incentive to get the group organized, and protected against outsiders. They refused to adopt the community model that was signified in the interface. They creatively used MSN Groups as a complementary tool, supporting the activities of a group defining itself as a "society". Throughout this initial period, then, SPEM clearly adopted the society F2F model, and there was no significant interference of the technologies they adopted on the activities they were engaged in.

However, Period 2 is marked by an extensive interference of technology in SPEM's social life. Because of technical problems with InForum, the group owner decided to create a forum in Yahoo! Groups. This dispersion turned into a major issue for the group. During this period, SPEM was using InForum, MSN Groups and Yahoo!Groups concomitantly. There was still uncertainty about the possibility of a complete migration to Yahoo!Groups, although there was a tendency to gradually concentrate SPEM activities in this space. As can be seen in the message below, members had doubts about how they would function as a group in the future.

"I can ask some questions, can't I? Are we all going to move there [Yahoo!Groups], and this space here [InForum] will be closed down? Will we be allowed to be in both places? (...) Have we solved our issues or created problems?"

During this period, the number of messages exchanged by SPEM members increased significantly, and their content was mainly about how members related to technology. Messages conveyed essentially two sub-categories of meanings: nostalgic feelings about their former space and feelings of loss and confusion in the new space. Here is a message from the group owner himself, posted at InForum:

"Subject: WHERE IS EVERYBODY??? Hi, I just came here to tell you how things are going in Yahoo. I see that all have moved there or given up. If you are still here, waiting, I have to say that:(...) it's all a matter of time to adapt and learn. If you haven't moved yet, we're waiting for you."

He calls the group's attention to the fact that a little learning effort is required to use Yahoo!Groups and that with time the technology will become familiar to everybody ("it's all a matter of time do adapt and learn"). Expressions of nostalgic feelings at this stage could perhaps be explained by the users' resistance to change. However, further evidence shows that there is yet another reason behind nostalgic feelings. Namely, members were used to interacting with MSN groups, and MSN groups and Yahoo!Groups look very much alike. For those who knew how to use MSN groups, Yahoo!Groups should have seemed easy (and familiar). So, unless we 
consider SPEM's sociability models in both systems aspects of the users' nostalgig feelings remain unexplained.

As previously mentioned, in MSN groups SPEM members seemed to ignore the designer's metacommunication. They were a society whose members used MSN as a complementary tool. At Yahoo!Groups, however, they started to work as a community, in consistence with the designer's metacommunication. Various messages express the fear to loose happiness and friendship. Even members who were used to post many messages at MSN groups (and therefore had skills to use the system) clearly express the melancholy caused by the migration: "Hi everyone, I hope we will be happy here ... as happy as we were there." Ambiguity and resistance seem to hide more than the need of learning more sophisticated features of a new system. It seems to be a matter of choice: should SPEM be a society (according to user's sociability model) or a community (in consistence with the designer's sociablity model)?

After this period of ambiguity, in March 2004, when Period 3 started, the creator of SPEM decided for a complete migration to Yahoo!Groups. The moderator wrote:

"About the forums: Inforum - is paid for till mar/04 (...) When this date expires, the forum will depend exclusively on Insite, who provides InForum services. I know that if they don't discontinue [the forum] there will always be someone there, other than me. Yahoo Brasil - was the best free forum option I found in the Internet because here: 1) They don't have as many problems in peak hours;2) It is possible to send almost instantly, via e-mail, everything that is in the forum, as long as one requests this at registration time; 3) There are many services available, like: polls, databases, chat, and others. And, best of all, its all 'gratis'! As for me, I am also trying to adapt to Yahoo, but I prefer the InForum/SPEM design. But I find Yahoo's services better than all other free Internet options that I have been trying."

Thus, technological issues, allied to the personal choices made by the moderator, seemed to have altered the sociability of SPEM. They turned from a society model into a community model. Nevertheless, the transition caused some loss. Various SPEM members dropped out, as message below shows:

"Subject: Jade says good bye and thanks to the Gang

My beloved friends! (this is my good bye). I want to thank you all for your kindness (...) through all this time we've been together (...) However, because I haven't been able to adapt to the new place I have asked to un-register. (...) I will keep with MSN and my email addresses for those who want to keep in touch online. (...) If you have something to tell me, do so... but I will only reply to your individual addresses, and no longer here or in Yahoo. I'm leaving taking my memories in my bag!"

In March 2006, two years after the migration, we saw that whereas in 2004 SPEM had some 500 members, now they were down to approximately 300, an important indication that various members did not adapt to the community model encoded in the technology, embraced by those who stayed. Another prime indication of the change is that the community sign made its way into the discourse of SPEM members: on their homepage, SPEM define themselves as a "virtual community". In January 2007, we saw that the community model has been consolidated. In line with this kind of model, the number of group members decreased to about 2/3 (208 members), policies were clearly defined and stated, and group membership required approval from the 
moderator. The number of posted messages is still good (about 100 per month), and the content indicates a high level of intimacy among members.

\subsection{Designers and Users Together at Interaction Time}

Further analysis of the results above led us to conclude that there were three kinds of signs affecting SPEM's life online. First, there were signs referring to their own lives and experience with MS, and to their feelings and opinions about other things, including their attitude toward technology. We called these user signs. Second, there were signs referring to design decisions and the features and behavior of each one of the three technologies. These were typically interface signs. They were encoded by technology designers, and users have sometimes learned them, sometimes ignored them, sometimes they didn't notice them, and sometimes they rejected or misinterpreted them. These signs carry the designers' values, their intent, the mark of their cultural background, and similarly subjective content. Lastly, there were signs imposed by computation itself, like the fact that every computable entity must have a unique symbolic identification (hence the need for names and ID's). This kind of sign may be in striking contrast with the semiosis of a non-institutionalized group. In previous work [5] we have discussed some social consequences of the limits imposed by computation signs. For the purposes of this paper, however, the most important finding with respect to this case study was the clear contrast between the designers' and users' signs that made their respective F2F sociability models explicit. From a semiotic engineering perspective designers communicate their vision to users at interaction time, and this vision includes their F2F model. Users, in their turn, have their own sociability models. At interaction time designers and users negotiate meanings related to these models, and the success of such negotiation will dictate the history of groups online.

\section{Discussion and Conclusion}

We believe that semiotic engineering has a relevant contribution to make for the study of $\mathrm{CMC}$ with respect to the migration of $\mathrm{F} 2 \mathrm{~F}$ to online communication. It calls the attention of researchers to the importance of consciously and systematically reflecting upon the underlying sociability models that are encoded in CMC technology. As seen with SPEM, these models are signified consciously or unconsciously by designers, in the systems interface. And users give strong evidence that such signs get into their semiosis, thus affecting their own sociability models and practices.

At interaction time, each group of users: (i) carries their own F2F sociability models to the online environment; (ii) interprets and reacts to the designers' communication about the F2F sociability model(s) they have encoded into the technology; and (iii) negotiates different sociability models, making conscious and/or unconscious judgments and decisions about the possibilities of using the technology.

Regarding (i), semiotic engineering suggests that there cannot be an ideal and universal sociability model for CMC. Users always bring with them different models and, therefore, personal reactions to different kinds of CMC environments will always vary. Some users will adapt quickly, others will never adapt, but there will always be 
a negotiation between technologically-encoded sociability models, on the one hand, and diverse culturally-inherited sociability models, on the other. Negotiation can be initiated because both are based on human (designers' and users') F2F social relations. But the success of negotiation will largely depend on the designers' awareness of what can be negotiated, why, and how. So, it we should beware of predictive models and universal solutions for online sociability. At best, we should seek for widely-applicable knowledge-generating methods to support CMC design. This alternative has the advantage to circumvent the embarrassing singularity of design cases where received heuristics and guidelines are not followed, but the design product is nevertheless evaluated as satisfactory by its users. In the SPEM case, we saw that the community loved the old discussion forum, which compared to MSN or Yahoo!Groups was precarious, according to established HCI design practices. The same situation was reported by Maloney-Krichmar and Preece [10], in their study of an online health community.

Regarding (ii), semiotic engineering explains that systems interfaces can signify things that designers are not even aware of having consciously expressed. In group settings, group semiosis can considerably amplify the semiotic effect of interface signs, as we have seen in our case study. Semiotic engineering calls the designers attention to the fact that users will interpret not only the signs that they have intentionally designed and encoded in the interface, but also those signs that they (designers) have unconsciously signified in the technology. The greater the designers' awareness of their own social values about F2F sociability models, and of how these relate to the users' sociability models, the greater the chances of successful negotiations at interaction time. The necessary mechanisms to support the users' choices will more likely be in place, and ready to be used.

Regarding (iii), through a comprehensive characterization of metacommunication, semiotic engineering encourages designers to spell out their metacommunication message at design time. They are stimulated to think of all static and dynamic signs, along with explicit explanatory signs directly expressing design rationale, which users should have access to in order to resolve potential disparities between their vision of sociability online, and the designers'. Careful and intentional selection of signs to communicate design intent will instigate a rich variety of computer-mediated communication between designers and users.

This is an interesting possibility because it allows us to examine CMC in greater detail, framing technological and natural signs, delivered by machines and humans alike, under a uniform set of concepts that constitute the semiotic engineering ontology. The theory amplifies the explicit knowledge of technology producers and researchers about what communication is, and the infinite variety of signification alternatives that users and designers may choose to communicate what they mean to and about each other. Thus the design process itself becomes a rich and sophisticated intellectual activity, very distant from a mere selection and application of existing solutions that will predictably work in previously known use situations.

In this paper we have presented a case study to illustrate how F2F sociability models are communicated and interpreted over CMC. However, the semiotic engineering ontology and the epistemic resources that we have used in an analytic process may perfectly be used for reflection at design time. The introduction of the designer's discourse as a specific stance of sign types in CMC allows us to detect 
subjective and cultural values incorporated to design patterns. Thus the message to CMC developers is that they may improve the quality of their products by: reflecting carefully about their context(s) of use, and the F2F sociability models that future users embrace; thinking critically about their own sociability models, rationalizing as many aspects of them as possible before they start encoding sociability models into the technology; and designing powerful negotiation mechanisms for users to understand the technology, adapt it to their purposes, and eventually use it to compose wider social spaces with technologies that suit their needs better in some particular respect.

Acknowledgments. Clarisse de Souza thanks CNPq for continuing support to her research. Clarissa Barbosa thanks CNPq and CAPES for her Ph.D. scholarships. All authors thank Marcelo Morita for facilitating this study, for his cooperation.

\section{References}

[1] Baudrillard, J.: The Vital Illusion. Columbia University Press, New York (2000)

[2] Bauman, Z.: Community: seeking safety in an insecure World. Blackwell Publishers, Malden, MA (2001)

[3] Castells, M.: The Internet galaxy: reflections on the Internet, business, and society. Oxford University Press, Oxford, New York (2001)

[4] Denzin, N.K., Lincoln, Y.S.(org.): Handbook of Qualitative Research, 2nd edn. Sage Publications, Thousand Oaks, CA (2000)

[5] de Souza, C.S., et al.: Compulsory institutionalization: investigating the paradox of computer-supported informal social processes. Interacting With Computers 16(4), 635656 (2004)

[6] de Souza, C.S.: The semiotic engineering of human-computer interaction. The MIT Press, Cambridge, MA (2005)

[7] de Souza, C.S., et al.: The Semiotic Inspection Method. In: VII Simpósio sobre Fatores Humanos em Sistemas Computacionais, IHC'2006, Natal, RN (2006). Anais do VII Simpósio sobre Fatores Humanos em Sistemas Computacionais. Porto Alegre, RS: SBC, vol. 1, pp. 148-157

[8] Dourish, P.: Where the action is. The MIT Press, Cambridge, MA (2001)

[9] Eco, U.: A theory of semiotics. Indiana University Press, Bloomington, IN (1976)

[10] Maloney-Krichmar, D., Preece, J.: A multilevel analysis of sociability, usability, and community dynamics in an online health community. ACM Transactions on ComputerHuman Interact 12(2), 201-232 (2005)

[11] Nicolaci-da-Costa, A.M., et al.: Como conhecer usuários através do Método de Explicitação do Discurso Subjacente (MEDS). In: Anais do VI Simpósio sobre Fatores Humanos em Sistemas Computacionais, pp. 47-56. SBC, Porto Alegre (2004)

[12] Nisbet, R.A.: The Sociological Tradition. Basic Books, New York (1966)

[13] Norman, D.A.: Emotional Design: why we Love (or hate) everyday things. Basic Books, N.Y. (2004)

[14] Peirce, C.S.: The Essential Peirce: Selected Philosophical Writings. In: Houser, N., Kloesel, C. (eds.), vol. 1, 2, Indiana University Press, Bloomington, IN (1992-1998)

[15] Picard, R.: Affective computing. The MIT Press, Cambridge, MA (1998)

[16] Schön, D.A.: The reflective practitioner. Basic Books,Inc., N.Y. (1983)

[17] Searle, J.R.: Expression and meaning. Cambridge University Press, Cambridge (1979)

[18] Winograd, T., Flores, F.: Understanding computers and cognition. Addison-Wesley, New York, NY (1986) 